\title{
Globular Clusters in the Local Group
}

\author{
Eva K. Grebel \\ Astronomisches Rechen-Institut, Zentrum für Astronomie der Universität Heidelberg, \\ Mönchhofstr. 12-14, 69120 Heidelberg, Germany \\ email: grebel@ari.uni-heidelberg.de
}

\begin{abstract}
Only twelve of the $>76$ Local Group galaxies contain globular clusters, showing a broad range of specific frequencies. Here we summarize the properties of these globular cluster systems. Many host galaxies contain very old globulars, but in some globular cluster formation may have been delayed. An age range of several Gyr is common. Except for the inner regions of the spirals, old globular clusters tend to be metal-poor. Increasingly, light element variations and hints of multiple stellar populations are being found also in extragalactic globulars. There is ample evidence for globular cluster accretion from dwarfs onto massive galaxies, but its magnitude has yet to be quantified. Caution is needed to avoid overinterpreting indirect evidence.
\end{abstract}

Keywords. Galaxy: globular clusters: general, Galaxy: halo, galaxies: star clusters, Local Group, galaxies: dwarfs, Magellanic Clouds, galaxies: abundances, galaxies: evolution, stellar content

\section{Introduction}

Star clusters are observed in all but the least massive Local Group galaxies (e.g. Grebel 2002). These clusters span a wide range of ages, metallicities, masses, and radii. Star clusters that can in principle survive for a Hubble time as bound stellar systems are called 'globular clusters'. For a review on globular cluster formation, see, e.g. Kruijssen (2014). Star clusters may dissolve due to their internal dynamical evolution, in particular because of relaxation processes. External influences such as dynamical friction and varying tidal fields (caused, e.g. by disk or bulge passages in their parent galaxy) may also lead to their gradual erosion, emphasizing the role of cluster environment. A cluster's survival depends on its mass and density and on its host galaxy's gravitational potential as well as the cluster's distance from and orbit in its host galaxy (e.g. Gnedin \& Ostriker 1997). Considering proper-motion-based orbits, Dinescu et al. (1999) find that the destruction rates due to internal two-body relaxation are higher than those due to tidal shocks.

Yet we still call an old star cluster currently undergoing disruption, such as Palomar 5 (Odenkirchen et al. 2001, 2002, Rockosi et al. 2002), a globular cluster. More generally, we may consider clusters that are plausibly massive and compact enough to survive as bound stellar aggregates for a Hubble time in the absence of destructive external or internal influences to be globular clusters. In fact, it is believed that many of the globular clusters that once formed were since destroyed by external gravitational effects. Indeed, a number of Galactic globular clusters show evidence for early stages of tidal disruption (e.g. Andreuzzi et al. 2001, Odenkirchen \& Grebel 2004, Grillmair \& Johnson 2006, Jordi \& Grebel 2010, Chun et al. 2010, Niederste-Ostholt et al. 2010, Kunder et al. 2014).

On the other hand, we do not usually call clusters much younger than $\sim 9$ Gyr globular clusters. Hence we consider the Small Magellanic Cloud (SMC) to contain only one true globular cluster (Glatt et al. 2008a), although many of its prominent intermediate-age clusters are massive enough to pass as likely globular candidates (see Glatt et al. 2008b, 2011). For the purpose of this review, we follow the convention of requiring an old age. 
There are additional properties that seem to be common to possibly all (Galactic) globular clusters, for instance, the lack of dark matter (e.g. Jordi et al. 2009; Frank et al. 2012; Sollima et al. 2012; but see Ibata et al. 2013). An important characteristic common to the majority of globular clusters are light element abundance variations (e.g. Kraft 1994; Harbeck et al. 2003a, 2003b; Gratton et al. 2004; Kayser et al. 2008), and/or the presence of multiple stellar populations (Gratton, Carretta, \& Bragaglia 2012). While massive old open clusters may overlap in age and even structural properties with lowmass, younger globular clusters, the absence of light element anticorrelations and of multiple populations appears to be a unique identifying property of open clusters (e.g. de Silva et al. 2009; Carretta et al. 2010a; Bragaglia et al. 2014; McLean et al. 2015). Such abundance information, however, is not yet available for most nearby extragalactic clusters and can thus not be used as a defining or distinguishing feature in this review. Conversely, as light element abundance anticorrelations seem to occur only in globular clusters, surveying field stars for such anomalies allows one to constrain the contribution of dissolved globular clusters to the Galactic field populations. These studies suggest that at least $17 \%$ of the present-day stellar mass observed as Galactic halo field stars has a globular cluster origin (Martell \& Grebel 2010; Martell et al. 2011).

Apart from light element abundance inhomogeneities, most globular clusters are fairly homogeneous in their heavy elements including iron (Carretta et al. 2009) with only very small star-to-star variations (e.g. Ivans et al. 1999, 2001; Cohen 2011; Yong et al. 2013) of $\sim 0.03$ to $0.05 \mathrm{dex}$ in $[\mathrm{Fe} / \mathrm{H}]$. The few globular clusters that are not mono-metallic include the most massive Galactic globular cluster, $\omega$ Centauri, which is believed to be the stripped nucleus of a former dwarf galaxy, the globular cluster at the center of the Sagittarius dwarf spheroidal (dSph) galaxy, M54, as well as a few other globulars (e.g. M2, M22, Terzan 5, NGC 5824). For details, see, for instance, Kraft (1994), Da Costa et al. (2009, 2014), Ferraro et al. (2009), Marino et al. (2009), Johnson \& Pilachowski (2010), Carretta et al. (2010b), Origlia et al. (2013), Yong et al. (2014), Massari et al. (2014), and Milone et al. (2015). For several of these unusual objects a (stripped) nuclear star cluster origin has been proposed to explain their internal range in metallicities. Those globular clusters with heavy element abundance spreads for which orbits have been determined seem to have originated from independent progenitors (Casetti-Dinescu et al. 2013).

Globular clusters differ from similarly luminous dwarf galaxies by their typically much more compact sizes with half-light radii of less than $10 \mathrm{pc}$ (although the recently discovered so-called extended globular clusters are more diffuse; see Huxor et al. 2005), by their apparent lack of dark matter (e.g. Gilmore et al. 2007a), by (usually) being mono-metallic systems (while even low-mass dwarf galaxies show appreciable abundance spreads; e.g. Grebel 1997, 2000; Harbeck et al. 2001; Shetrone et al. 2001; Grebel et al. 2003; Koch et al. 2006, 2007a, 2007b; Norris et al. 2008; Adén et al. 2009, 2011; Kirby et al. 2011; Simon et al. 2011), and by not following a metallicity-luminosity relation. Globular clusters and dSph galaxies lie on different fundamental planes, but globular clusters and ultra-compact dwarf (UCD) galaxies show some overlap (Tollerud et al. 2011).

The separation of globular clusters from dSphs in mass-radius-luminosity space is primarily driven by whether objects have the mass-to-light ratios typical for old stellar populations $(\sim 3$, as globular clusters do), or whether they are dark-matter-dominated (Tollerud et al. 2011; see also Zaritsky et al. 2006 and Misgeld \& Hilker 2011). The UCD locus, with which the brightest globular clusters overlap, tilts away from the pure mass-follows-light relation (Tollerud et al. 2011). Kinematic studies of UCDs show that some have globular-cluster-like mass-to-light ratios suggesting that they are indeed very massive star clusters (e.g. Mieske et al. 2008; Frank et al. 2011), while others have higher 
mass ratios supporting a "galaxian" nature (e.g. stripped nuclei or lower-mass compact ellipticals; see, for instance, Brodie et al. 2011 and Forbes et al. 2014).

\section{Differences and commonalities of the M31, M33, and Galactic globular cluster systems}

M31, the dominant spiral galaxy in the Local Group, also has the richest globular cluster system. More than 700 globular cluster candidates have been identified in M31 and its surroundings so far (see Huxor et al. 2014; Galleti et al. 2004, and the regularly updated Revised Bologna Catalogue†). In contrast, in the Milky Way only about 160 globular clusters are known (see Harris 1996 and the continuing updates of the Harris catalog $\ddagger)$.

The luminosity functions of the globular cluster systems of the Milky Way and M31 have similar median absolute luminosities of $M_{V}=-7.3$ and -7.6 , respectively (Huxor et al. 2014). When considering only M31 globular clusters at projected galactocentric distances larger than $30 \mathrm{kpc}$, the resulting luminosity function shows a secondary maximum at lower luminosities $(\sim-5.5)$. This resembles the bimodal values in the sparsely sampled globular cluster luminosity function of Sagittarius (van den Bergh 1998). This as well as the fact that many of the remote globular clusters of M31 are associated with the stellar tidal streams found around this galaxy suggests an accretion origin for the outer halo globular cluster population (Mackey et al. 2010, 2013; Huxor et al. 2014).

More metal-rich Milky Way and M31 globular clusters tend to be located at smaller galactocentric distances, although there is a range of metallicities at any distance (Wang \& Ma 2013). More metal-rich globulars typically have smaller half-light radii. Globular cluster half-light radii and tidal radii increase with galactocentric distance in both galaxies (McLaughlin \& van der Marel 2005; Barmby et al. 2007; Huxor et al. 2011; Wang \& Ma 2013) as expected in scenarios of environmentally driven cluster survivability (e.g. Gnedin \& Ostriker 1997). Faint globular clusters show a range of half-light radii, whereas the brighter ones tend to be primarily compact (van den Bergh 1996; Huxor et al. 2014).

The most metal-rich globular clusters $([\mathrm{Fe} / \mathrm{H}]>-0.5 \mathrm{dex})$ in $\mathrm{M} 31$ are mainly located in the bulge region in the innermost $5 \mathrm{kpc}$. They follow the steeply rising $\mathrm{H}$ I rotation curve of the bulge (Lee et al. 2008; Galleti et al. 2009). Even the more widely distributed metalpoor globular clusters $([\mathrm{Fe} / \mathrm{H}]<-1.0 \mathrm{dex})$ exhibit a clear rotational pattern although their velocity dispersion is much larger (Galleti et al. 2009). This may be the signature of a rotating, yet pressure-supported halo (Lee et al. 2008). A subset of M31 globular clusters shows X-ray emission and follows the rotation of the disk (Lee et al. 2008).

The most metal-rich globular clusters $([\mathrm{Fe} / \mathrm{H}]>-0.8 \mathrm{dex})$ in the Milky Way are almost exclusively situated within the inner $10 \mathrm{kpc}$ around the Galactic center and are concentrated in the bulge region. They show a flattened distribution and are believed to belong to the bulge and the thick disk (e.g. Mackey \& Gilmore 2004a). For a growing number of Galactic globular clusters, orbit determinations are becoming available. Some globular clusters (metal-rich ones but also a few metal-poor ones) were found to belong kinematically to the thick disk or to the bar (Casetti-Dinescu et al. 2010). Others show clear classical hot halo kinematics with extended, highly eccentric and in part retrograde orbits (Dauphole et al. 1996; Dinescu et al. 1999). The so-called "young" halo globular clusters (Zinn et al. 1993) with predominantly red horizontal branches, but $[\mathrm{Fe} / \mathrm{H}]<-1$ dex stand out as a hot population with mainly high-energy, large-size, and highly eccentric

$\dagger$ http://www.bo.astro.it/M31/

$\ddagger$ http://physwww.mcmaster.ca/ ${ }^{\text {harris/mwgc.dat }}$ 
orbits in the outer regions of the Milky Way, consistent with a possible accretion origin (e.g. Dinescu et al. 1999). These objects have the widest spatial distribution and are found out to distances of more than $100 \mathrm{kpc}$ (e.g. Mackey \& Gilmore 2004a). The radial mass density profile of the surviving old halo globular clusters, on the other hand, follows the expected profile of the cold protogalactic gas (Parmentier \& Grebel 2005).

Most bulge, disk, and old halo globular clusters in the Milky Way have core radii $<3$ pc, while globular clusters of the "young" halo and in Galactic satellites may exhibit core radii up to $\sim 20$ pc (Mackey \& Gilmore 2004a). This similarity in structural properties along with the eccentric and in part retrograde orbits may support a dwarf galaxy origin for "young halo" globulars. Typical compact globular clusters in the Milky Way and M31 have half-light radii of up to 5 pc, but the diffuse, "extended globular clusters" described by Huxor et al. (2005) can reach half-light radii of 30 pc. Such objects are found at galactocentric distances from $\sim 15$ to $\sim 150 \mathrm{kpc}$ around M31 and our Galaxy. Extended globular clusters just seem to sample the upper end of the globular cluster size distribution while behaving like regular, old, metal-poor globulars in all other aspects (Huxor et al. 2014). Da Costa et al. (2009b) suggested that there may be two modes of cluster formation, one leading to compact and one to extended clusters, with extended globular clusters forming (and surviving) preferentially in lower tidal field environments such as dwarf galaxies (see also Elmegreen 2008). In plots of the radius-luminosity relation, extended globular clusters begin to fill the gap between "normal" globular clusters and ultra-faint dSphs, raising interesting questions about the presence of dark matter or gradual dynamical or tidal dissolution (Misgeld \& Hilker 2011; Huxor et al. 2011).

The designation "young" for a subset of halo clusters mentioned earlier describes their location in a diagram of horizontal branch type versus metallicity: these globular clusters have a lower metallicity than expected from their horizontal branch type; i.e., one would expect a bluer horizontal branch at their metallicity. Hence a second parameter must be responsible for their horizontal branch morphology. When plotting horizontal branch isochrones in the above diagram, these clusters appear to be $\sim 1$ to 3 Gyr younger than the oldest globular clusters (e.g. Lee et al. 1994; Keller et al. 2012). However, the locus in the horizontal branch type - metallicity diagram is not only determined by age. Other factors, such as helium abundance or central density, also play a role. Nonetheless, age dating based on deep color-magnitude diagrams (CMDs) often corroborates a younger age (e.g. Stetson et al. 1999; Rey et al. 2001; Dotter et al. 2010; VandenBerg et al. 2013).

Keller et al. (2012) find that the "young halo" globular clusters at Galactocentric distances larger than $10 \mathrm{kpc}$ lie close to a plane that is very similar to the plane encompassing the Galactic satellites, supporting the suggestion that they were accreted. VandenBerg et al. (2013) argue that the age-metallicity relation of Galactic globular clusters splits into two branches, which are offset by 0.6 dex. The more metal-rich branch shows disklike kinematics, while the more metal-poor one has halo kinematics. Leaman et al. (2013) find good agreement between the metal-poor branch and the age-metallicity relation of Local Group irregular galaxies. They propose that a large fraction of the halo globular cluster system was accreted from such galaxies. - In summary, there are many different lines of arguments favoring accretion of part of the Galactic globular cluster system.

Among luminous M31 globulars extended blue horizontal branches are common, suggestive of multiple stellar populations (Perina et al. 2012). Taking the locus in the horizontal branch type - metallicity diagram as a proxy for age, Perina et al. (2012) find that M31 globular clusters at projected galactocentric distances of 10 to $100 \mathrm{kpc}$ are $\sim 0.4$ dex more metal-rich at a given horizontal branch type than their Galactic counterparts. This suggests that they typically formed 1 to 2 Gyr later than even the "young" Galactic halo globulars if helium abundance variations can be neglected - an intriguing 
systematic difference. For M31's globular clusters there are generally no sufficiently deep CMD data available for an independent, accurate age determination. However, for one globular cluster Hubble Space Telescope (HST) imaging reaching $m_{V} \sim 30.5$ mag was obtained, which provides data extending below the main-sequence turn-off. The resulting isochrone-based age is 2 to 3 Gyr younger than for typical Galactic globular clusters at that metallicity and horizontal branch morphology (Brown et al. 2004).

We have less detailed information about the globular clusters of M33. Surveys of the star cluster system of this smallest Local Group spiral reveal a large number of young and intermediate-age clusters and a distribution of integrated colors similar to that of the Large Magellanic Cloud (LMC) (e.g. Sarajedini \& Mancone 2007). Thus far these surveys suffer from spatial and luminosity incompleteness, but also from the difficulty to distinguish genuine star clusters from other extended sources such as background galaxies or asterisms in crowded regions (e.g. Park \& Lee 2007; Zloczewski \& Kaluzny 2009).

Moreover, age estimates based on resolved CMDs obtained from HST data are available only for a subset of cluster candidates (e.g. Cockcroft et al. 2011; Zloczewski \& Kaluzny 2009; Park et al. 2009; San Roman et al. 2009), and these are typically too shallow to permit an accurate age dating of old cluster candidates. Globular cluster candidates have thus been identified mainly via integrated colors, e.g. from their overlap with the locus of Galactic globular clusters in two-color diagrams (e.g. Ma 2012, 2013) and age-dated via their multi-color spectral energy distributions (e.g. Fan \& de Grijs 2014). The Fan \& de Grijs catalog contains 75 globular cluster candidates older than 10 Gyr and approximately 17 candidates located in the 8-10 Gyr range within the uncertainties of their age estimates. In addition, there are at least six outer halo globular cluster candidates within projected radii of 10 to $50 \mathrm{kpc}$ around M33 (Cockcroft et al. 2011). This comparatively low number may indicate that the outer halo of M33 was significantly stripped during past encounters with M31 or that M33 experienced a much more quiescent accretion history (Cockcroft et al. 2011). At least one outer halo globular cluster in M33 is an extended cluster similar to the ones found in the outskirts of the Milky Way and M31 (Stonkutè et al. 2008).

The "specific frequency" of a galaxy is its number of globular clusters, $N_{\mathrm{GC}}$, normalized by unit parent galaxy luminosity; $S_{N}=N_{\mathrm{GC}} \cdot 10^{0.4\left(M_{V}+15\right)}$ (Harris \& van den Bergh 1981). If all globular cluster candidates in M33 are genuine globulars, then M33's specific frequency is 2 to 2.7 , implying a rather rich globular cluster system for a low-mass disk galaxy. The $S_{N}$ values of M31 and of the Milky Way are $>2.3$ and $\sim 0.7$, respectively. Given their incompleteness and uncertain detections, these numbers are likely to change.

\section{Globular clusters in Local Group dwarf spheroidal galaxies}

The Local Group contains a large number of metal-poor, gas-deficient dSphs, most of which are satellites of the two massive spirals (see Grebel et al. 2003 for details). In recent years, many new, very faint dSph companions of the Milky Way and M31 were discovered (e.g. Zucker et al. 2004, 2006a, 2006b, 2007; Belokurov et al. 2006; Martin et al. 2006; Walsh et al. 2007). A comprehensive tabulation of the key properties of Local Group dwarf galaxies including the recent discoveries is given in McConnachie (2012) $\dagger$ and its continued updates. Only the two most luminous dSphs, Sagittarius and Fornax, host globular clusters.

The Sagittarius dSph galaxy, which is currently merging with the Milky Way, contains five high-, four moderate, and two low-confidence globular cluster members (Law

$\dagger$ https://www.astrosci.ca/users/alan/Nearby_Dwarfs_Database.html 
\& Majewski 2010). Assuming that Sagittarius' total luminosity was $M_{V}=-15$ prior to its disruption, its resulting specific frequency is 5 to 9 depending on how many globular clusters are associated with it (Law \& Majewski 2010). The specific frequency of Sagittarius (henceforth Sgr) is much higher than that of more luminous galaxies, where $S_{N}$ scatters around 1 (Harris et al. 2013). Higher values of $S_{N}$ are typically only found in the most massive elliptical galaxies (such as $\mathrm{cD}$ or brightest cluster galaxies) as well as in low-mass, gas-deficient galaxies such as dSphs and nucleated dwarf ellipticals (Harris et al. 2013; Grebel 2002).

As noted by Da Costa \& Armandroff (1995), Sgr contributes both "old halo" as well as "young halo" globular clusters to the Milky Way - a cautionary point that is important to remember when discussing globular cluster accretion. The Sgr globular cluster candidates follow a fairly narrow age-metallicity relation, which can be well approximated by a simple closed-box model with a continuous star formation rate (Forbes \& Bridges 2010).

The spatial coincidence of the very massive, very luminous globular cluster M54 with the center of Sgr indicates that M54 may be the nucleus of this dSph galaxy (Sarajedini \& Layden 1995), possibly akin to what is seen in more massive nucleated dwarf ellipticals (e.g. Lisker et al. 2006, 2007). However, more recent studies revealed that Sgr has a metal-rich nucleus with a flat velocity dispersion profile that is independent of the metalpoor M54 with its steeply rising velocity dispersion (Monaco et al. 2005; Bellazzini et al. 2008). This suggests that M54 may have formed elsewhere in Sgr and eventually sank to its center via dynamical friction (Bellazzini et al. 2008). We thus see the actual nucleus and M54 in superposition; a coincidence that would be impossible to disentangle in more distant galaxies. M54 has a metallicity of $[\mathrm{Fe} / \mathrm{H}] \sim-1.6$ with an extended tail towards higher metallicities, similar to the metallicity spread found in $\omega$ Centauri (Carretta et al. 2010c). Just like other globular clusters, it shows a $\mathrm{Na}-\mathrm{O}$ anticorrelation, which is not seen in the stars attributed to the Sgr nucleus (Carretta et al. 2010c).

The Fornax dSph galaxy contains five metal-poor globular clusters, four of which are located in its outer regions, while one of them (globular cluster \#4 in the naming convention of Hodge 1961) lies close to its center. This fourth globular cluster is more metal-rich than the others (Larsen et al. 2012) and approximately 3 Gyr younger (Buonanno et al. 1999), while the other four are indistinguishable in age and as old as the oldest Galactic globular clusters (Buonanno et al. 1998). Fornax' globular cluster \#1 is the most metal-poor globular cluster known to date $([\mathrm{Fe} / \mathrm{H}]=-2.5$ dex, Letarte et al. 2006). The specific frequency of Fornax is $\sim 22.8$, the highest value of any Local Group galaxy.

The metal-poor, ancient, roughly coeval globular clusters \#3 and \#5 show pronounced differences in their horizontal branch morphologies (Smith et al. 1996), again adding a cautionary note to the use of horizontal branch indices as age indicators. If Fornax were to be accreted by the Milky Way, it, too, would contribute both genuinely old as well as "young halo" globular clusters. Nitrogen abundance variations and horizontal branch morphologies in its four old globular clusters suggest the presence of multiple stellar populations, just like in Galactic globulars (D'Antona et al. 2013; Larsen et al. 2014a).

Oh et al. (2000) pointed out that due to dynamical friction it is unexpected to find Fornax' four old globular clusters in the outer regions of this galaxy instead of in the center, forming a merged, very massive nucleus. Oh et al. (2000) suggest tidal heating and significant tidal mass loss of Fornax as a possible mechanism preventing the clusters' orbital decay. Goerdt et al. (2006) and Cole et al. (2012) argue that a cored dark matter halo (as opposed to a cuspy cold dark matter halo) extending to the positions of the globular clusters would likewise prevent this. Strigari et al. (2006), on the other hand, challenge the notion of a very large core. Generally though, cored dark matter halos seem to reproduce best the kinematic data of galaxies of a range of different Hubble types (e.g. 
Gilmore et al. 2007b; Donato et al. 2009; Salucci et al. 2012). For dSphs like Fornax, a cuspy profile was found to be excluded at high significance (Walker \& Peñarrubia 2011).

Several other Galactic dSphs show dynamically cold substructures, which may also share similar metallicities (e.g. Kleyna et al. 1998, 2004; Walker et al. 2006; Battaglia et al. 2011). If real (see the cautionary remarks of Ural et al. 2010), these structures may be the remnants of dissolved old star clusters. Just like the old globular clusters in Fornax, the longevity and survival of these potential remnants support cored dark matter halos (e.g. Lora et al. 2012a, 2012b, 2013). We note that initially cuspy cold dark matter halos can evolve into cored dark matter profiles due to the redistribution of matter via star formation and feedback (e.g. Mashchenko et al. 2006; Pasetto et al. 2010).

\section{Globular clusters in Local Group dwarf ellipticals}

M31 is the only galaxy in the Local Group that has dwarf elliptical (dE) companions (e.g. Grebel 1997; 2001). While its three dE satellites NGC 147, NGC 185, and NGC 205 all host globular clusters, its compact elliptical companion M32 apparently (and unexpectedly, given its luminosity) does not (e.g. van den Bergh 2000; Rudenko et al. 2009). NGC 205 and NGC 185 show prominent intermediate-age populations as well as recent star formation, gas, and dust, whereas NGC 147 is largely old and gas-deficient (see Grebel 1999 and references therein). NGC 205 and M32 both contain conspicuous nuclei.

Globular cluster candidates in NGC 205 were first identified by Hubble (1932). Followup studies, particularly metallicity and age determinations from integrated spectra and shallow HST CMDs, suggest that Hubble I, II, IV, VI, VII, and VIII are genuine old globular clusters with estimated ages ranging from $7-11$ Gyr $( \pm 2 \mathrm{Gyr})$ and $[\mathrm{Fe} / \mathrm{H}]$ from -1.1 to -2.0 dex (Da Costa \& Mould 1988; Sharina et al. 2006; Colucci \& Bernstein 2011). The enhanced $[\alpha / \mathrm{Fe}]$ ratios in the old globular clusters indicate high star formation rates at early times (Colucci \& Bernstein 2011). The resulting $S_{N}$ of NGC 205 is 1.7.

The first globular cluster candidates in NGC 147 and NGC 185 were discovered by Baade (1944). More candidates were added in the following decades, including recent detections by Sharina \& Davoust (2009) in NGC 147 and by Veljanoski et al. (2013) in both dEs. The new additions are in part much fainter than the early detections and tend to be located at larger distances from the centers of the galaxies. Contamination by clusters in the disk or spheroid of M31 seen in superposition are an issue. Many of the globular cluster candidates in both dEs have been analyzed using various methods based on integrated spectra, radial velocities, and integrated photometry (e.g. Da Costa \& Mould 1988; Sharina et al. 2006; Sharina \& Davoust 2009; Veljanoski et al. 2013). The inferred ages and metallicities differ considerably in some cases (as also for NGC 205). Some of the candidates were found to be too young to be considered bona fide globular clusters, although the age estimates are rather uncertain and can be affected by the surrounding integrated field star light (Veljanoski et al. 2013). Regardless of the method, most globular clusters are found to be metal-poor $([\mathrm{Fe} / \mathrm{H}]<-1.2 \mathrm{dex})$. Sharina et al. (2006) point out that many of the globular cluster candidates in the three dEs overlap with the Galactic "young halo" locus in the metallicity - horizontal-branch-type diagram.

NGC 147 may contain up to ten globular clusters, but two of these (the metal-rich Hodge IV, which exhibits a somewhat discrepant radial velocity, and SD-10) may not be genuine globulars (Sharina \& Davoust 2009; Veljanoski et al. 2013). NGC 185 hosts seven promising globular cluster candidates. Accounting for the uncertainties, Veljanoski et al. (2013) estimated the specific frequencies to be $8 \pm 2$ for NGC 147 and $5.5 \pm 0.5$ for NGC 185. These values follow the trend in $S_{N}$ in other dEs of similar luminosity. 


\section{Globular clusters in Local Group (dwarf) irregular galaxies}

The LMC is the most luminous, most massive irregular galaxy in the Local Group. It contains at least 15 globular clusters older than $\sim 10$ Gyr (e.g. Mackey \& Gilmore 2004b; Baumgardt et al. 2013) and one slightly younger candidate (ESO 121-SC03; age $\sim 8.9$ Gyr; Xin et al. 2008). The LMC's specific frequency is 0.6. It also hosts numerous intermediate-age and young populous clusters (e.g. Girardi et al. 1995; Hunter et al. 2003; Glatt et al. 2010), but shows an as yet unexplained lack of clusters with ages of about 5 to 9 Gyr (Olszewski et al. 1991). Many more clusters than expected from chance superpositions appear to be double or multiple with similarly old components (Bhatia \& Hatzidimitrou 1988; Bhatia et al. 1991; Dieball \& Grebel 2000; Dieball et al. 2002).

The oldest globular clusters in the LMC are indistinguishable in age from the oldest Galactic, Sagittarius, and Fornax globular clusters (see Olsen et al. 1998; Johnson et al. 1999; Grebel \& Gallagher 2004). Intriguingly, the SMC's only globular cluster, NGC 121, is 2-3 Gyr younger (Glatt et al. 2008a). Moreover, and in contrast to the LMC (and the Milky Way), the SMC formed massive star clusters throughout its history (e.g. Da Costa \& Hatzidimitriou 1998; Glatt et al. 2008b), which is unexpected especially if the two Clouds were indeed close to and interacted with each other for most of their lifetime.

Despite their small age range, the LMC globulars cover a variety of horizontal branch morphologies and metallicities (Olszewski et al. 1991; Olsen et al. 1998; Mackey \& Gimore 2004b; Johnson et al. 2006; Mucciarelli et al. 2010). High-resolution spectra of individual red giants show that the old globular clusters in the LMC show similar $\mathrm{Na}-\mathrm{O}$ anticorrelations as Galactic globular clusters (Mucciarelli et al. 2009; Mateluna et al. 2012). Abundances from integrated spectra reveal an $[\alpha / \mathrm{Fe}]$ spread in old LMC globular clusters of similar metallicity, suggesting that the interstellar medium was not well mixed at the time of their formation (Colucci et al. 2012). Several old LMC globular clusters show much lower $[\alpha / \mathrm{Fe}]$ ratios than comparable Galactic globular clusters (Johnson et al. 2006; Mateluna et al. 2012), which poses an interesting challenge for scenarios where the early accretion of massive satellites contributed substantially to the build-up of the Galactic halo and its globular clusters (e.g. De Lucia \& Helmi 2008; Leaman et al. 2013). On the other hand, for even more metal-poor old LMC field stars, $[\alpha / \mathrm{Fe}]$ is in very good agreement with Galactic halo stars of the same metallicity (Haschke et al. 2012a).

With only one genuine, albeit slightly younger globular cluster it is difficult to analyze the SMC $\left(S_{N} \sim 0.1\right)$ in the same manner. In any case, this galaxy shows a range of cluster metallicities at any given age (Glatt et al. 2008b; Cignoni et al. 2013), which implies that its interstellar gas was not well mixed throughout its history. The SMC's star clusters do not show any preferred rotation (e.g. Da Costa \& Hatzidimitriou 1998), consistent with the highly perturbed structure of this interacting dwarf irregular galaxy (e.g. Haschke et al. 2012b). In contrast, even the old LMC globular clusters follow disk as opposed to halo kinematics (Schommer et al. 1992; Grocholski et al. 2006).

Only two of the "isolated" dwarf irregular galaxies in the Local Group host globular clusters. In NGC 6822 eight globular cluster candidates have been identified, including five extended, diffuse clusters and three compact and luminous clusters (Hwang et al. 2011; Huxor et al. 2013). Assuming that they are all genuine globular clusters, the resulting $S_{N} \sim 3$. In its central regions, NGC 6822 also contains a number of intermediate-age and young star clusters. The old, extended globular clusters appear to lie roughly along the major axis of the old stellar halo, almost perpendicular to the elongated $\mathrm{H}$ i distribution of NGC 6822 (Hwang et al. 2011; Huxor et al. 2013). Just like in the SMC some of the globular clusters have high ellipticities. Integrated spectroscopy suggests that the globular clusters are metal-poor with $[\mathrm{Fe} / \mathrm{H}]$ from -1.5 to -2.5 and ages between $\sim 8$ to 
Table 1. Globular cluster systems of Local Group galaxies

\begin{tabular}{|l|l|c|c|c|l|}
\hline Galaxy & Type & $\boldsymbol{M}_{V}$ & $\boldsymbol{N}_{\mathrm{GC}}$ & $\boldsymbol{S}_{N}$ & Remarks \\
\hline M31 & SA(s)b & -21.2 & $>700$ & $>2.3$ & Rotation, [Fe/H] gradient, on avg. younger; accretion \\
Milky Way & SBbc & -20.9 & $\sim 160$ & $\sim 0.7$ & [Fe/H] gradient, Na-O anticorrelation, accretion \\
M33 & SA(s)cd & -18.9 & $75-98$ & $2-2.7$ & Outer halo stripped? \\
Sgr & dSph(t) & $\sim-15.0$ & $5-11$ & $5-9$ & "Young" \& "old halo" GCs; Na-O anticorrelation \\
For & dSph & -13.1 & 5 & 22.8 & "Young" \& "old halo" GCs; multiple pop.? [Fe/H] grad. \\
NGC 205 & dE5, N & -16.4 & $\sim 6$ & 1.7 & "Young" \& "old halo" GCs; $\alpha /$ Fe] enhanced \\
NGC 185 & dE3 & -15.6 & $\sim 7$ & $\sim 4$ & "Young" \& "old halo" GCs; solar $[\alpha /$ Fe] \\
NGC 147 & dE5 & -15.1 & $8-10$ & $\sim 7-9$ & Near-solar [ $\alpha /$ Fe]? \\
LMC & SB(s)m & -18.5 & $15-16$ & 0.6 & Rotation, "young" \& "old halo", Na-O, $[\alpha /$ Fe] spread \\
SMC & SB(s)mp & -17.1 & 1 & 0.1 & Younger than oldest MW \& LMC GCs, high ellipticity \\
NGC 6822 & IB(s)m & -16.0 & 8 & 3 & Some GCs: high ellipt., one w. solar $[\alpha /$ Fe]. No rotation \\
WLM & IB(s)m & -14.4 & 1 & 1.7 & Na-O anticorr.? [ $\alpha /$ Fe] enhanced, high ellipticity \\
\hline
\end{tabular}

Notes: Column 1: galaxy name; column 2: galaxy classification (mainly from the NASA/IPAC Extragalactic Database or NED; ned.ipac.caltech.edu); column 3: absolute V-band magnitude (see Grebel 2002, Grebel et al. 2003); column 4: number of globular clusters (caution: incompleteness, false detections!); column 5: specific frequency (same warning); column 6: some of the special properties of a galaxy's globular cluster system (see relevant chapters of this review for details and references). MW stands for Milky Way, GC for globular cluster.

14 Gyr (Cohen \& Blakeslee 1998; Hwang et al. 2014). The old cluster Hubble VII shows solar $[\mathrm{Ca} / \mathrm{Fe}$ (Colucci \& Bernstein 2011) and is close to the center of NGC 6822, while the other old, metal-poor globular clusters have galactocentric distances $>2 \mathrm{kpc}$ (Hwang et al. 2014). They do not rotate with the disk of NGC 6822 (Hwang et al. 2014).

In WLM, the faintest dIrr in the Local Group known to contain a globular cluster, only one such object is known, resulting in a relatively high specific frequency of 1.7. WLM's globular cluster is unusual in several respects: It is very luminous, highly eccentric yet very compact (Stephens et al. 2006), and lies in the outer regions of WLM at a projected distance of about $400-500$ pc from the main body of the dIrr. Only one other, but very young luminous star cluster has been found in WLM so far, located in its central regions, and there are no known populous intermediate-age star clusters (Hodge et al. 1999).

A deep HST CMD and integrated spectra suggest that the globular cluster in WLM is old (> $12 \mathrm{Gyr}$ ) and metal-poor with inferred metallicities ranging from $\sim-1.5$ to $\sim-2$ dex (see Hodge et al. 1999; Stephens et al. 2006; Colucci \& Bernstein 2011; Larsen et al. 2014b). Stephens et al. (2006) find a fairly high central velocity dispersion of $\sim 10 \mathrm{~km} \mathrm{~s}^{-1}$ for the globular cluster and no evidence of rotation. They suggest that a high velocity dispersion anisotropy is the most likely reason for the cluster's high ellipticity. Larsen et al. (2014b) show that WLM's globular cluster has similar abundance patterns as the old, metal-poor globular clusters in the Milky Way and in Fornax, including $\alpha$ element enhancement (see also Colucci et al. 2011). While integrated spectra do not allow one to measure light element abundance anticorrelations, Larsen et al. interpret the cluster's high $[\mathrm{Na} / \mathrm{Fe}]$ ratio as a possible indication of a second generation of star formation.

\section{Concluding remarks}

Out of the more than 76 galaxies of the Local Group, only twelve host one or more globular clusters. The globular cluster systems exhibit a bewildering range of richness and specific frequencies, while the lack of globular clusters in other galaxies of similar type and luminosity is not yet understood. Many globular clusters were only discovered in recent years though. Their census is likely to continue to increase in the coming years, particularly with respect to faint, extended clusters. Most host galaxies contain very old globular clusters (as well as some that are up to a few Gyr younger), but in some galaxies 
(such as the SMC and perhaps M31) all globular clusters seem to be systematically younger. The reasons for such a possibly delayed cluster formation are still unclear.

There is ample direct and indirect evidence of globular cluster accretion from dwarf galaxies onto M31 and the Milky Way, but its magnitude remains to be quantified. As dwarf galaxies may contribute both "young" and "old" halo globular clusters, as well as globulars with or without $[\alpha / \mathrm{Fe}]$ enhancement, one needs to beware of too simplistic conclusions. Globulars in dwarf galaxies often show large core radii and/or high ellipticities. The preferred occurrence of globular clusters with large core radii in the outer halos of massive galaxies may imply an accretion origin and/or suggest that more extended clusters form and survive more easily in environments with lower tidal fields. $\mathrm{Na}-\mathrm{O}$ anticorrelations or extended horizontal branches in globular clusters in several dwarf galaxies indicate that multiple stellar populations may be a global property of globular clusters. Finally, globular clusters can help to constrain the dark halo properties of their hosts.

\section{References}

Adén, D., et al. 2009, A\&\&A, 506, 1147

Adén, D., et al. 2011, A\&A, 525, A153

Andreuzzi, G., et al. 2001, A\&BA, 372, 851

Baade, W. 1944, ApJ, 100, 147

Barmby, P., McLaughlin, D. E., Harris, W. E., Harris, G. L. H., \& Forbes, D. A. 2007, AJ, 133, 2764

Battaglia, G., Tolstoy, E., Helmi, A., Irwin, M., Parisi, P., Hill, V., \& Jablonka, P. 2011, MNRAS, 411, 1013

Baumgardt, H., Parmentier, G., Anders, P., \& Grebel, E. K. 2013, MNRAS, 430, 676

Bellazzini, M., et al. 2008, AJ, 136, 1147

Belokurov, V., et al. 2006, ApJL, 647, L111

Bhatia, R. K. \& Hatzidimitriou, D. 1988, MNRAS, 230, 215

Bhatia, R. K., Read, M. A., Hatzidimitriou, D., \& Tritton, S. 1991, A\&AS, 87, 335

Bica, E., Bonatto, C., Dutra, C. M., \& Santos, J. F. C. 2008, MNRAS, 389, 678

Brodie, J. P., Romanowsky, A. J., Strader, J., \& Forbes, D. A. 2011, AJ, 142, 199

Brown, T. M., et al. 2004, ApJL, 613, L125

Buonanno, R., et al. 1998, ApJL, 501, L33

Buonanno, R., et al. 1999, AJ, 118, 1671

Carretta, E., Bragaglia, A., Gratton, R., D’Orazi, V., \& Lucatello, S. 2009, A\&A, 508, 695

Carretta, E., et al. 2010a, A\&A, 516, A55

Carretta, E., et al. 2010b, ApJL, 714, L7

Carretta, E., et al. 2010c, A\&A, 520, A95

Casetti-Dinescu, D. I., Girard, T. M., Korchagin, V. I., van Altena, W. F., \& López, C. E. 2010, $A J, 140,1282$

Casetti-Dinescu, D. I., et al. 2013, AJ, 146, 33

Chun, S.-H., et al. 2010, AJ, 139, 606

Cignoni, M., et al. 2013, ApJ, 775, 83

Cockcroft, R., et al. 2011, ApJ, 730, 112

Cohen, J. G. 2011, ApJL, 740, L38

Cohen, J. G. \& Blakeslee, J. P. 1998, AJ, 115, 2356

Cole, D. R., Dehnen, W., Read, J. I., \& Wilkinson, M. I. 2012, MNRAS, 426, 601

Colucci, J. E. \& Bernstein, R. A. 2011, in A Universe of Dwarf Galaxies, EAS Publications Series 48, eds. M. Koleva, P. Prugniel, \& I. Vauglin (EDP Sciences: Paris), 275

Colucci, J. E., Bernstein, R. A., Cameron, S. A., \& McWilliam, A. 2012, ApJ, 746, 29

Da Costa, G. S. \& Mould, J. R. 1988, ApJ, 334, 159

Da Costa, G. S. \& Armandroff, T. E. 1995, AJ, 109, 2533

Da Costa, G. S. \& Hatzidimitriou, D. 1998, AJ, 115, 1934 
Da Costa, G. S., Held, E. V., Saviane, I., \& Gullieuszik, M. 2009a, ApJ, 705, 1481

Da Costa, G. S., Grebel, E. K., Jerjen, H., Rejkuba, M., \& Sharina, M. E. 2009b, AJ, 137, 4361

Da Costa, G. S., Held, E. V., \& Saviane, I. 2014, MNRAS, 438, 3507

D'Antona, F., et al. 2013, MNRAS, 434, 1138

Dauphole, B., Geffert, M., \& Colin, J., et al. 1996, A\&A, 313, 119

De Lucia, G. \& Helmi, A. 2008, MNRAS, 391, 14

de Silva, G. M., Gibson, B. K., Lattanzio, J., \& Asplund, M. 2009, A\&\&A, 500, L25

Dieball, A. \& Grebel, E. K. 2000, A\& A, 358, 897

Dieball, A., Müller, H., \& Grebel, E. K. 2002, A\& $A$, 391, 547

Dinescu, D. I., Girard, T. M., \& van Altena, W. F. 1999, AJ, 117, 1792

Donato, F., et al. 2009, MNRAS, 397, 1169

Dotter, A., et al. 2010, ApJ, 708, 698

Elmegreen, B. G. 2008, ApJ, 672, 1006

Fan, Z. \& de Grijs, R. 2014, ApJS, 211, 22

Ferraro, F. R., et al. 2009, Nature, 462, 483

Forbes, D. A. \& Bridges, T. 2010, MNRAS, 404, 1203

Forbes, D. A., et al. 2014, MNRAS, 444, 2993

Frank, M. J., et al. 2011, MNRAS, 414, L70

Frank, M. J., et al. 2012, MNRAS, 423, 2917

Galleti, S., Federici, L., Bellazzini, M., Fusi Pecci, F., \& Macrina, S. 2004, A\&A, 416, 917

Galleti, S., Bellazzini, M., Buzzoni, A., Federici, L., \& Fusi Pecci, F. 2009, A\&̋A, 508, 1285

Gilmore, G., et al. 2007a, Nuclear Physics B Proceedings Supplements, 173, 15

Gilmore, G., et al. 2007, ApJ, 663, 948

Girardi, L., Chiosi, C., Bertelli, G., \& Bressan, A. 1995, A\&A, 298, 87

Glatt, K., et al. 2008a, AJ, 135, 1106

Glatt, K., et al. 2008b, $A J, 136,1703$

Glatt, K., et al. 2009, AJ, 138, 1403

Glatt, K., Grebel, E. K., \& Koch, A. 2010, A\&A, 517, A50

Glatt, K., et al. 2011, AJ, 142, 36

Gnedin, O. Y. \& Ostriker, J. P. 1997, ApJ, 474, 223

Goerdt, T., Moore, B., Read, J. I., Stadel, J., \& Zemp, M. 2006, MNRAS, 368, 1073

Gratton, R., Sneden, C., \& Carretta, E. 2004, ARA\&A, 42, 385

Gratton, R. G., Carretta, E., \& Bragaglia, A. 2012, A\&ARv, 20, 50

Grebel, E. K. 1997, Reviews in Modern Astronomy, 10, 29

Grebel, E. K. 1999, in The Stellar Content of Local Group Galaxies, IAU Symp. 192, eds. P. Whitelock \& R. Cannon (San Francisco: ASP), 17

Grebel, E. K. 2000, in Star Formation from the Small to the Large Scale, 33rd ESLAB Symposium, ESA SP 445, eds. F. Favata, A.A. Kaas, \& A. Wilson (Noordwijk: ESA), p. 87

Grebel, E. K. 2001, Astrophysics and Space Science Supplement, 277, 231

Grebel, E. K. 2002, in Extragalactic Star Clusters, IAU Symp. 207, eds. D. Geisler, E. K. Grebel, \& D. Minniti (San Francisco: ASP), 94

Grebel, E. K., Gallagher, J. S., \& III, Harbeck, D. 2003, AJ, 125, 1926

Grebel, E. K. \& Gallagher, J. S. 2004, ApJL, 610, L89

Grillmair, C. J., \& Johnson, R. 2006, ApJL, 639, L17

Grocholski, A. J., Cole, A. A., Sarajedini, A., Geisler, D., \& Smith, V. V. 2006, AJ, 132, 1630

Harbeck, D., et al. 2001, AJ, 122, 3092

Harbeck, D., Smith, G. H., \& Grebel, E. K. 2003a, AJ, 125, 197

Harbeck, D., Smith, G. H., \& Grebel, E. K. 2003b, A\&\&A, 409, 553

Harris, W. E. 1996, AJ, 112, 1487

Harris, W. E. \& van den Bergh, S. 1981, $A J, 86,1627$

Harris, W. E., Harris, G. L. H., \& Alessi, M. 2013, ApJ, 772, 82

Haschke, R., et al. 2012a, AJ, 144, 88

Haschke, R., Grebel, E. K., \& Duffau, S. 2012b, AJ, 144, 107

Hodge, P. W. 1961, AJ, 66, 83 
Hodge, P. W., Dolphin, A. E., Smith, T. R., \& Mateo, M. 1999, ApJ, 521, 577

Hubble, E. 1932, ApJ, 76, 44

Hunter, D. A., Elmegreen, B. G., Dupuy, T. J., \& Mortonson, M. 2003, AJ, 126, 1836

Huxor, A. P., et al. 2005, MNRAS, 360, 1007

Huxor, A. P., et al. 2011, MNRAS, 414, 770

Huxor, A. P., Ferguson, A. M. N., Veljanoski, J., Mackey, A. D., \& Tanvir, N. R. 2013, MNRAS, 429, 1039

Huxor, A. P., et al. 2014, MNRAS, 442, 2165

Hwang, N., et al. 2011, ApJ, 738, 58

Hwang, N., et al. 2014, ApJ, 783, 49

Ibata, R., et al. 2013, MNRAS, 428, 3648

Ivans, I. I., et al. 1999, $A J, 118,1273$

Ivans, I. I., et al. 2001, $A J, 122,1438$

Johnson, J. A., Bolte, M., Stetson, P. B., Hesser, J. E., \& Somerville, R. S. 1999, ApJ, 527, 199

Johnson, J. A., Ivans, I. I., \& Stetson, P. B. 2006, ApJ, 640, 801

Johnson, C. I. \& Pilachowski, C. A. 2010, ApJ, 722, 1373

Jordi, K., et al. 2009, AJ, 137, 4586

Jordi, K. \& Grebel, E. K. 2010, A $₫ A, 522$, A71

Kayser, A., Hilker, M., Grebel, E. K., \& Willemsen, P. G. 2008, A\& A, 486, 437

Keller, S. C., Mackey, D., \& Da Costa, G. S. 2012, ApJ, 744, 57

Kirby, E. N., Lanfranchi, G. A., Simon, J. D., Cohen, J. G., \& Guhathakurta, P. 2011, ApJ, 727,78

Kleyna, J. T., Geller, M. J., Kenyon, S. J., Kutz, M. J., \& Thorstensen, J. R. 1998, ApJ, 115, 2359

Kleyna, J. T., Wilkinson, M. I., Evans, N. W., \& Gilmore G. 2004, MNRASL, 354, L66

Koch, A., et al. 2006, AJ, 131, 895

Koch, A., et al. 2007a, ApJ, 657, 241

Koch, A., et al. 2007b, AJ, 133, 270

Kraft, R. P. 1994, PASP, 106, 553

Kruijssen, J. M. D. 2014, Classical and Quantum Gravity, 31, 244006

Kunder, A., et al. 2014, A\& A, 572, A30

Larsen, S. S., Brodie, J. P., \& Strader, J. 2012, A\&A, 546, A53

Larsen, S. S., Brodie, J. P., Grundahl, F., \& Strader, J. 2014a, ApJ, 797, 15

Larsen, S. S., Brodie, J. P., Forbes, D. A., \& Strader, J. 2014b, A\& A, 565, A98

Law, D. R. \& Majewski, S. R. 2010, ApJ, 718, 1128

Leaman, R., VandenBerg, D. A., \& Mendel, J. T. 2013, MNRAS, 436, 122

Lee, Y.-W., Demarque, P., \& Zinn, R. 1994, ApJ, 423, 248

Lee, M. G., et al. 2008, ApJ, 674, 886

Letarte, B., et al. 2006, A\& A, 453, 547

Lisker, T., Glatt, K., Westera, P., \& Grebel, E. K. 2006, AJ, 132, 2432

Lisker, T., Grebel, E. K., Binggeli, B., \& Glatt, K. 2007, ApJ, 660, 1186

Lora, V., Magaña, J., Bernal, A., Sánchez-Salcedo, F. J., \& Grebel, E. K. 2012a, JCAP, 2, 011

Lora, V., Just, A., Sánchez-Salcedo, F. J., \& Grebel, E. K. 2012, ApJ, 757, 87

Lora, V., Grebel, E. K., Sánchez-Salcedo, F. J., \& Just, A. 2013, ApJ, 777, 65

Ma, J. 2012, AJ, 144, 41

Ma, J. 2013, $A J, 145,88$

Mackey, A. D. \& Gilmore, G. F. 2004a, MNRAS, 355, 504

Mackey, A. D. \& Gilmore, G. F. 2004b, MNRAS, 352, 153

Mackey, A. D., Wilkinson, M. I., Davies, M. B., \& Gilmore, G. F. 2008, MNRAS, 386, 65

Mackey, A. D., et al. 2010, ApJL, 717, L11

Mackey, A. D., et al. 2013, MNRAS, 429, 281

Marino, A. F., et al. 2009, AESA, 505, 1099

Martell, S. L. \& Grebel, E. K. 2010, A\&A, 519, A14

Martell, S. L., Smolinski, J. P., Beers, T. C., \& Grebel, E. K. 2011, A\&̛A, 534, A136 
Martin, N. F., et al. 2006, MNRAS, 371, 1983

Mashchenko, S., Couchman, H. M. P., \& Wadsley, J. 2006, Nature, 442, 539

Massari, D., et al. 2014, ApJ, 795, 22

Mateluna, R., et al. 2012, A\&A, 548, A82

McConnachie, A. W. 2012, AJ, 144, 4

McLaughlin, D. E. \& van der Marel, R. P. 2005, ApJS, 161, 304

McLean, B. T., De Silva, G. M., \& Lattanzio, J. 2015, MNRAS, 446, 3556

Mieske, S., et al. 2008, A\&A A 487, 921

Milone, A. P., et al. 2015, MNRAS, 447, 931

Misgeld, I. \& Hilker, M. 2011, MNRAS, 414, 3699

Monaco, L., Bellazzini, M., Ferraro, F. R., \& Pancino, E. 2005, MNRAS, 356, 1396

Mucciarelli, A., Origlia, L., Ferraro, F. R., \& Pancino, E. 2009, ApJL, 695, L134

Mucciarelli, A., Origlia, L., \& Ferraro, F. R. 2010, ApJ, 717, 277

Niederste-Ostholt, M., et al. 2010, MNRAS, 408, L66

Norris, J. E., et al. 2008, ApJL, 689, L113

Odenkirchen, M., et al. 2001, ApJL, 548, L165

Odenkirchen, M., et al. 2002, AJ, 124, 1497

Odenkirchen, M., \& Grebel, E. K. 2004, in Satellites and Tidal Streams, ASP Conf. Ser. 327, eds. F. Prada \& D. Martínez-Delgado (San Francisco: ASP), 284

Oh, K. S., Lin, D. N. C., \& Richer, H. B. 2000, ApJ, 531, 727

Olsen, K. A. G., Hodge, P. W., \& Mateo, M., et al. 1998, MNRAS, 300, 665

Olszewski, E. W., Schommer, R. A., Suntzeff, N. B., \& Harris, H. C. 1991, AJ, 101, 515

Origlia, L., et al. 2013, ApJL, 779, L5

Rey, S.-C., Yoon, S.-J., Lee, Y.-W., Chaboyer, B., \& Sarajedini, A. 2001, AJ, 122, 3219

Rudenko, P., Worthey, G., \& Mateo, M. 2009, AJ, 138, 1985

Park, W.-K. \& Lee, M. G. 2007, AJ, 134, 2168

Park, W.-K., Park, H. S., \& Lee, M. G. 2009, ApJ, 700, 103

Parmentier, G., \& Grebel, E. K. 2005, MNRAS, 359, 615

Pasetto, S., Grebel, E. K., Berczik, P., Spurzem, R., \& Dehnen, W. 2010, A\&A, 514, A47

Perina, S., et al. 2012, A\& $A, 546$, A31

Rockosi, C. M., et al. 2002, AJ, 124, 349

Salucci, P., et al. 2012, MNRAS, 420, 2034

San Roman, I., Sarajedini, A., Garnett, D. R., \& Holtzman, J. A. 2009, ApJ, 699, 839

Sarajedini, A., \& Layden, A. C. 1995, AJ, 109, 1086

Sarajedini, A. \& Mancone, C. L. 2007, AJ, 134, 447

Schommer, R. A., Suntzeff, N. B., Olszewski, E. W., \& Harris, H. C. 1992, AJ, 103, 447

Sharina, M. E., Afanasiev, V. L., \& Puzia, T. H. 2006, MNRAS, 372, 1259

Sharina, M. \& Davoust, E. 2009, A\&\&A, 497, 65

Shetrone, M. D., Côté, P., \& Sargent, W. L. W. 2001, ApJ, 548, 592

Simon, J. D., et al. 2011, ApJ, 733, 46

Smith, E. O., Neill, J. D., Mighell, K. J., \& Rich, R. M. 1996, AJ, 111, 1596

Sollima, A., et al. 2012, ApJ, 744, 196

Stetson, P. B., et al. 1999, AJ, 117, 247

Stephens, A. W., Catelan, M., \& Contreras, R. P. 2006, AJ, 131, 1426

Stonkutè, et al. 2008, AJ, 135, 1482

Strigari, L. E., et al. 2006, ApJ, 652, 306

Tollerud, E. J., Bullock, J. S., Graves, G. J., \& Wolf, J. 2011, ApJ, 726, 108

Ural, U., et al. 2010, MNRAS, 402, 1357

van den Bergh, S. 1996, AJ, 112, 2634

van den Bergh, S. 1998, ApJL, 505, L127

van den Bergh, S. 2000, The Galaxies of the Local Group, Cambridge Astrophysics Series 35 (Cambridge: Cambridge Univ. Press)

VandenBerg, D. A., Brogaard, K., Leaman, R., \& Casagrande, L. 2013, ApJ, 775, 134

Veljanoski, J., et al. 2013, MNRAS, 435, 3654 
Walker, M. G., Mateo, M., Olszewski, E. W., Pal, J. K., Sen, B., \& Woodroofe, M. 2006, ApJL, 642, L41

Walker, M. G. \& Peñarrubia, J. 2011, ApJ, 742, 20

Walsh, S. M., Jerjen, H., \& Willman, B. 2007, ApJL, 662, L83

Wang, S. \& Ma, J. 2013, AJ, 146, 20

Xin, Y., Deng, L., de Grijs, R., Mackey, A. D., \& Han, Z. 2008, MNRAS, 384, 410

Yong, D., et al. 2013, MNRAS, 434, 3542

Yong, D., et al. 2014, MNRAS, 441, 3396

Zaritsky, D., Gonzalez, A. H., \& Zabludoff, A. I. 2006, ApJ, 638, 725

Zinn, R. 1993, in The Globular Cluster-Galaxy Connection, ASP Conf. Ser. 48, eds. G. H. Smith \& J. P. Brodie (ASP: San Francisco), p. 38

Zloczewski, K. \& Kaluzny, J. 2009, Acta Astronomica, 59, 47

Zucker, D. B., et al. 2004, ApJL, 612, L121

Zucker, D. B., et al. 2006a, ApJL, 643, L103

Zucker, D. B., et al. 2006b, ApJL, 650, L41

Zucker, D. B., et al. 2007, ApJL, 659, L21 